\title{
ON SOME CHARACTERIZATION PROBLEMS CONNECTED WITH STOCHASTIC INTEGRALS*
}

\author{
BY \\ ROBERT LUGANNANI AND JOHN B. THOMAS \\ Princeton University
}

I. Introduction. Let $y_{1}$ and $y_{2}$ be distinct linear combinations of the independent random variables $x_{1}, \cdots, x_{n}$. Various relationships between $y_{1}$ and $y_{2}$ have been used to characterize normality of the $x_{i}$ 's. Among these are independence $[1,2]$, restrictions on conditional moments [3-6], and restrictions on the distributions [7, 8]. Certain of these results have been extended to infinitely many random variables $[9,10]$ and to a special class of stochastic integrals [11,12]. Concern in the integral case is with conditions that distinguish the Brownian motion process. A discussion of much of the existing work on these characterization problems and a number of additional references can be found in [13].

In the present paper we are concerned with a class of stochastic integrals that are more general than those considered by earlier authors. Various characterizations of independence and normality are obtained for a family of these integrals and the relationship between normality and independence is examined. In addition to extending some of the earlier results, the characterization problems for the stochastic integrals are of interest in the study of an important class of processes-the linear processes. These processes include as special cases a number of well-known processes used as models of physical phenomena [14]. As an application we consider three different series expansions for the linear processes; it is shown that the random coefficients in these expansions can be independent only if the processes are normal.

II. Preliminaries: stochastic integrals. Throughout, $R$ will denote a Euclidean space of fixed but arbitrary dimension, and integration with respect to a probability measure will be denoted by $E$.

A random measure $X$ is a family of random variables with the properties:

(1) To every bounded Borel set $B$ of $R$ there corresponds a random variable $X(B)$ with $E|X(B)|^{2}<\infty$.

(2) If $B_{1}, B_{2}, \cdots$ are disjoint Borel sets whose union $B$ is bounded then $X(B)=$ $X\left(B_{1}\right)+X\left(B_{2}\right)+\cdots$ where the series converges in quadratic mean.

The random measures considered in this paper are assumed to be real and to satisfy $E X(B)=0$ for every Borel set $B$.

A random measure has independent components if, for every collection of disjoint Borel sets $B_{1}, \cdots, B_{n}$, the random variables $X\left(B_{1}\right), \cdots, X\left(B_{n}\right)$ are mutually inde-

\footnotetext{
* Received July 10, 1969; revised version received February 18, 1970.
} 
pendent. If $X$ has independent components, the set function $V(B)=E|X(B)|^{2}$ is a Borel measure on $R$.

The random measure $X$ has stationary components if the joint distribution of the family $X\left(\tau+B_{1}\right), \cdots, X\left(\tau+B_{n}\right)$ does not depend upon $\tau$. In the case of independent components, stationarity is equivalent to requiring that $X(B)$ and $X(\tau+B)$ be identically distributed for every $B$ and every $\tau$. If $X$ has stationary components then $V$ is a Haar measure and is equal to Lebesgue measure on the Borel sets to within a nonnegative multiplicative constant.

Denote by $L_{2}(V)$ the Hilbert space of real-valued functions which are square integrable with respect to the measure $V$. For every $f \in L_{2}(V)$ the stochastic integral $\int_{R} f(s) X(d s)$ is defined as a limit in quadratic mean of sums formally associated with the integral [14].

Let $X$ be a random measure with independent components and, for every point $t$ with $V(\{t\})>0$, let $X(\{t\})$ be infinitely divisible. We will be concerned with the family of stochastic integrals

$$
y_{k}=\int_{R} f_{k}(s) X(d s), \quad k=1, \cdots, n
$$

where $f_{1}, \cdots, f_{n} \in L_{2}(V)$. For these integrals we have

$$
E y_{k}=0, \quad k=1, \cdots, n
$$

and

$$
E y_{i} y_{k}=\int_{R} f_{j}(s) f_{k}(s) V(d s), \quad j, k=1, \cdots, n .
$$

As a consequence of the restrictions imposed upon $X$, explicit expressions are available for the joint characteristic function of the random variables $y_{1}, \cdots, y_{n}$. Denoting this characteristic function by $\Phi\left(u_{1}, \cdots, u_{n}\right)$ we have [14]

$\Phi\left(u_{1}, \cdots, u_{n}\right)=\exp \left[\int_{R} \int_{-\infty}^{\infty}\left\{\exp \left[i \lambda \mathbf{u}^{T} \cdot \mathbf{f}(s)\right]-i \lambda \mathbf{u}^{T} \cdot \mathbf{f}(s)-1\right\} \frac{1}{\lambda^{2}} G(d s \times d \lambda)\right]$

where $\mathbf{u}$ and $\mathbf{f}(s)$ are the column vectors $\left(u_{1}, \cdots, u_{n}\right)^{T}$ and $\left(f_{1}(s), \cdots, f_{n}(s)\right)^{T}$, respectively, and the integrand is defined by continuity at $\lambda=0$. The function $G$ is a Borel measure on the product space $R \times(-\infty, \infty)$ and is uniquely determined by the random measure $X$ via the transform

$$
-\frac{d^{2}}{d u^{2}} \ln E \exp [i u X(B)]=\int_{B} \int_{-\infty}^{\infty} \exp [i \lambda u] G(d s \times d \lambda) .
$$

It is evident from (5) that

$$
V(B)=\int_{B} \int_{-\infty}^{\infty} G(d s \times d \lambda) .
$$

If a random measure has stationary components then the associated measure $G$ factors into the product of Lebesgue measure on the Borel sets of $R$ and a finite measure on $(-\infty, \infty)$.

Two important random measures are those characterized by normal laws and by 
Poisson laws. In the normal (Brownian motion) case

$$
\begin{aligned}
G(B \times A) & =V(B), & & \{0\} \in A \\
& =0, & & \{0\} \notin A,
\end{aligned}
$$

and in the Poisson case

$$
\begin{aligned}
G(B \times A) & =V(B), & & \{1\} \in A \\
& =0, & & \{1\} \notin A .
\end{aligned}
$$

The measure $G_{1}(B \times A)=G(B \times(A \cap\{0\}))$ is called the normal component of $G$. We shall also need the measure $G_{2}(B \times A)=G(B \times(A-\{0\}))$. Clearly $G=G_{1}+$ $G_{2}$. Corresponding to $G_{1}$ and $G_{2}$ we define the Borel measures

$$
V_{1}(B)=\int_{B} \int_{-\infty}^{\infty} G_{1}(d s \times d \lambda)
$$

and

$$
V_{2}(B)=\int_{B} \int_{-\infty}^{\infty} G_{2}(d s \times d \lambda),
$$

and obviously $V=V_{1}+V_{2}$. We shall have occasion to consider functions, $f(s)$, which are almost everywhere zero with respect to the measures $G_{2}$ or $V_{2}$. These conditions will be written as $f(s)=O\left[G_{2}\right]$ and $f(s)=O\left[V_{2}\right]$, respectively.

III. Characterizations of independence. Throughout the remainder of the paper, $\left\{y_{1}, \cdots, y_{n}\right\}$ is a family of random variables defined by (1).

THEOREM 1. In order that the random variables $y_{1}, \cdots, y_{n}$ be mutually independent it is necessary and sufficient that

$$
\int_{R} f_{j}(s) f_{k}(s) V(d s)=0, \quad j \neq k=1, \cdots, n
$$

and

$$
f_{i}(s) f_{k}(s)=O\left[V_{2}\right], \quad j \neq k=1, \cdots, n .
$$

Proof. Using the notation of (4) it is evident that $y_{1}, \cdots, y_{n}$ will be mutually independent if and only if for every $u_{1}, \cdots, u_{n}$

$$
\int_{R} \int_{-\infty}^{\infty}\left\{\exp \left[i \lambda \mathbf{u}^{T} \cdot \mathbf{f}(s)\right]-\sum_{k=1}^{n} \exp \left[i \lambda u_{k} f_{k}(s)\right]+(n-1)\right\} \frac{1}{\lambda^{2}} G(d s \times d \lambda)=0 .
$$

To establish the sufficiency, observe that for $n \geq 2$

$$
\begin{aligned}
& \left|\int_{R} \int_{-\infty}^{\infty}\left\{\exp \left[i \lambda \mathbf{u}^{r} \cdot \mathbf{f}(s)\right]-\sum_{k=1}^{n} \exp \left[i \lambda u_{k} f_{k}(s)\right]+(n-1)\right\} \frac{1}{\lambda^{2}} G(d s \times d \lambda)\right| \\
& \leq \sum_{j=2}^{n} \sum_{k=1}^{i-1}\left|u_{j} u_{k}\right|\left\{\left|\int_{R} f_{j}(s) f_{k}(s) V_{1}(d s)\right|+\int_{R}\left|f_{j}(s) f_{k}(s)\right| V_{2}(d s)\right\}
\end{aligned}
$$

where we have made use of the inequality

$$
\left|\exp \left[i \sum_{k=1}^{n} \xi_{k}\right]-\sum_{k=1}^{n} \exp \left[i \xi_{k}\right]+(n-1)\right| \leq \sum_{j=2}^{n} \sum_{k=1}^{i-1}\left|\xi_{i} \xi_{k}\right|
$$


which is valid for all real $\xi_{1}, \cdots, \xi_{n}$ and can be established by induction on $n$. It follows from (8) and (9) that both integrals on the right side of (11) are zero, so that (10) holds.

Turning to the necessity, it is clear that, because the random variables have zero mean and are independent,

$$
E y_{i} y_{k}=0, \quad j \neq k=1, \cdots, n
$$

which is equivalent to (8). To establish (9), note that (10) holds for any two random variables $y_{i}$ and $y_{k}$ with $j \neq k$. Thus

$$
\int_{R} \int_{-\infty}^{\infty}\left\{\exp \left[i \lambda u_{i} f_{i}(s)\right]-1\right\} \cdot\left\{\exp \left[i \lambda u_{k} f_{k}(s)\right]-1\right\} \frac{1}{\lambda^{2}} G(d s \times d \lambda)=0,
$$

and, by adding the four equations corresponding to the different combinations of $\pm u_{i}$ and $\pm u_{k}$, we obtain

$$
\int_{R} \int_{-\infty}^{\infty}\left\{1-\cos \lambda u_{j} f_{j}(s)\right\} \cdot\left\{1-\cos \lambda u_{k} f_{k}(s)\right\} \frac{1}{\lambda^{2}} G_{2}(d s \times d \lambda)=0 .
$$

This result holds for all $u_{i}$ and $u_{k}$ and, since the integrand is nonnegative and $\lambda=0$ is a point of $G_{2}$-measure zero, either $f_{i}(s)=0$ or $f_{k}(s)=0$ for every $s \in R$ with the possible exception of a set of $G_{2}$-measure zero; consequently

$$
\int_{R} \int_{-\infty}^{\infty}\left|f_{i}(s) f_{k}(s)\right| G_{2}(d s \times d \lambda)=\int_{R}\left|f_{i}(s) f_{k}(s)\right| V_{2}(d s)=0
$$

and $f_{i}(s) f_{k}(s)=0\left[V_{2}\right]$, completing the proof.

CoROLLARY. The random variables $y_{1}, \cdots, y_{n}$ are mutually independent if and only if they are pairwise independent.

The next result provides the basic characterization of independence in terms of moments.

THEOREM 2. For the independence of $y_{1}, \cdots, y_{n}$ it is necessary and sufficient that

$$
E y_{i} y_{k}=0, \quad j \neq k=1, \cdots, n
$$

and

$$
E y_{j}^{2} y_{k}^{2}=E y_{j}^{2} E y_{k}^{2}, \quad j \neq k=1, \cdots, n .
$$

Proof. The necessity is obvious. To establish the sufficiency we will show that the conditions of the previous theorem are satisfied. Since (8) and (14) are equivalent, only property (9) remains. Combining the relationship

$$
E y_{i}^{2} y_{k}^{2}=\int_{R} \int_{-\infty}^{\infty} f_{i}^{2}(s) f_{k}^{2}(s) \lambda^{2} G(d s \times d \lambda)+E y_{i}^{2} \cdot E y_{k}^{2}+2 E^{2} y_{i} y_{k}
$$

with the conditions of the theorem, we obtain

$$
\int_{R} \int_{-\infty}^{\infty} f_{j}^{2}(s) f_{k}^{2}(s) \lambda^{2} G(d s \times d \lambda)=\int_{R} \int_{-\infty}^{\infty} f_{j}^{2}(s) f_{k}^{2}(s) \lambda^{2} G_{2}(d s \times d \lambda)=0 .
$$

Since the integrand is nonnegative and $\lambda=0$ is a point of $G_{2}$-measure zero, it follows that $f_{i}(s) f_{k}(s)=O\left[G_{2}\right]$ or equivalently $f_{i}(s) f_{k}(s)=O\left[V_{2}\right]$, completing the proof.

If $y_{i}$ and $y_{k}$ are independent, it is clear that $E\left[y_{i} \mid y_{k}\right]=0$ and $E\left[y_{i}^{2} \mid y_{k}\right]=E y_{i}^{2}$. 
The next result shows that these regression properties are also sufficient for the independence of $y_{i}$ and $y_{k}$.

THEOREM 3. The random variables $y_{1}, \cdots, y_{\mathbf{n}}$ are mutually independent if and only if

$$
E\left[y_{i} \mid y_{k}\right]=0, \quad j \neq k=1, \cdots, n
$$

and

$$
E\left[y_{i}^{2} \mid y_{k}\right]=E y_{i}^{2}, \quad j \neq k=1, \cdots, n
$$

Proof. Since the necessity is obvious, we consider only the sufficiency. Using the hypotheses of the theorem, we have for $j \neq k$

$$
E y_{i} y_{k}=E y_{k} E\left[y_{i} \mid y_{k}\right]=0, \quad E y_{i}^{2} y_{k}^{2}=E y_{k}^{2} E\left[y_{i}^{2} \mid y_{k}\right]=E y_{i}^{2} \cdot E y_{k}^{2}
$$

and the independence follows from Theorem 2 .

IV. Characterizations of normality. In contrast to the earlier characterization problems, which have been concerned with distinguishing those random measures that are Brownian motions, we are concerned here with conditions which are equivalent to the joint normality of the random variables $y_{1}, \cdots, y_{n}$.

THEOREM 4. The random variables $y_{1}, \cdots, y_{n}$ are jointly normal if and only if

$$
f_{k}(s)=O\left[V_{2}\right], \quad k=1, \cdots, n .
$$

Proof. It is clear from (4) that $y_{1}, \cdots, y_{n}$ are jointly normal if and only if

$$
\int_{R} \int_{-\infty}^{\infty}\left\{\exp \left[i \lambda \mathbf{u}^{T} \cdot \mathbf{f}(s)\right]-\frac{1}{2}\left[i \lambda \mathbf{u}^{T} \cdot \mathbf{f}(s)\right]^{2}-i \lambda \mathbf{u}^{T} \cdot \mathbf{f}(s)-1\right\} \frac{1}{\lambda^{2}} G(d s \times d \lambda)=0 .
$$

Considering first the sufficiency, observe that the left side of (19) is bounded above by

$$
\begin{aligned}
\int_{R} \int_{-\infty}^{\infty}\left|\exp \left[i \lambda \mathbf{u}^{T} \cdot \mathbf{f}(s)\right]-\frac{1}{2}\left[i \lambda \mathbf{u}^{T} \cdot \mathbf{f}(s)\right]^{2}-i \lambda \mathbf{u}^{T} \cdot \mathbf{f}(s)-1\right| & \frac{1}{\lambda^{2}} G(d s \times d \lambda) \\
& \leq \int_{R}\left|\mathbf{u}^{T} \cdot \mathbf{f}(s)\right|^{2} V_{2}(d s) .
\end{aligned}
$$

But since $f_{k}(s)=O\left[V_{2}\right], k=1, \cdots, n$, it follows that the right side of $(20)$ is zero and (19) is established.

Turning to the necessity, note that the real part of (19) must be zero; that is,

$$
\begin{aligned}
0 & =\int_{R} \int_{-\infty}^{\infty}\left\{\cos \lambda \mathbf{u}^{T} \cdot \mathbf{f}(s)+\frac{1}{2}\left[\lambda \mathbf{u}^{T} \cdot \mathrm{f}(s)\right]^{2}-1\right\} \frac{1}{\lambda^{2}} G(d s \times d \lambda) \\
& =\int_{R} \int_{-\infty}^{\infty}\left\{\cos \lambda \mathbf{u}^{T} \cdot \mathrm{f}(s)+\frac{1}{2}\left[\lambda \mathbf{u}^{T} \cdot \mathrm{f}(s)\right]^{2}-1\right\} \frac{1}{\lambda^{2}} G_{2}(d s \times d \lambda) .
\end{aligned}
$$

Since the integrand is nonnegative and $\lambda=0$ is a point of $G_{2}$-measure zero, it follows that $\mathbf{u}^{T} \cdot \mathbf{f}(s)=O\left[G_{2}\right]$. Finally, noting that $\mathbf{u}$ is arbitrary, we have $f_{k}(s)=O\left[G_{2}\right]$ or, equivalently, $f_{k}(s)=O\left[V_{2}\right]$ for $k=1, \cdots, n$ and the proof is complete.

Corollary. The random variables $y_{1}, \cdots, y_{n}$ are jointly normal if and only if their univariate distributions are normal.

The normality of the random variables $y_{1}, \cdots, y_{n}$ can also be expressed in terms of their moments. We have 
THEOREM 5. For the random variables $y_{1}, \cdots, y_{n}$ to be jointly normal it is necessary and sufficient that

$$
E y_{k}^{4}=3 E^{2} y_{k}^{2}, \quad k=1, \cdots, n .
$$

Proof. The necessity is well known. To establish the sufficiency, observe that

$$
E y_{k}^{4}=\int_{R} \int_{-\infty}^{\infty} f_{k}^{4}(s) \lambda^{2} G(d s \times d \lambda)+3 E^{2} y_{k}^{2} .
$$

Combining this relation with (21) yields

$$
\int_{R} \int_{-\infty}^{\infty} f_{k}^{4}(s) \lambda^{2} G(d s \times d \lambda)=\int_{R} \int_{-\infty}^{\infty} f_{k}^{4}(s) \lambda^{2} G_{2}(d s \times d \lambda)=0,
$$

and the same reasoning used earlier shows that $f_{k}(s)=O\left[V_{2}\right], k=1, \cdots, n$. The joint normality then follows from Theorem 3.

If the underlying random measure $X$ is a Brownian motion, then every family of stochastic integrals defined in terms of $X$ is jointly normal. However the foregoing results indicate that for certain choices of the integrands $f_{1}, \cdots, f_{n}$, the corresponding random variables $y_{1}, \cdots, y_{n}$ may be normal without requiring that $X$ be a Brownian motion. Nevertheless it is of some interest to examine conditions under which normality of the stochastic integrals implies that $X$ is a Brownian motion. One such condition is given by

TheOREM 6. Let the measure $G$ factor into the product of a Borel measure on $R$ and a finite measure on $(-\infty, \infty)$. If at least one of the random variables $y_{1}, \cdots, y_{n}$ is nondegenerate and normally distributed then the underlying random measure $X$ is a Brownian motion.

Proof. According to the hypothesis we may write $G(B \times A)=V(B) F(A)$ where the measure $F$ satisfies $\int_{-\infty}^{\infty} F(d \lambda)=1$. Let $y_{k}$ be nondegenerate so that $f_{k}(s) \neq O[V]$. Since $y_{k}$ is normal, we have (from Theorem 4) $f_{k}(s)=O\left[V_{2}\right]$ where

$$
V_{2}(B)=\int_{B} \int_{-\infty}^{\infty} G_{2}(d s \times d \lambda)=V(B) \cdot[1-F(\{0\})] .
$$

Thus the only way in which the normality is compatible with the nondegeneracy is for $F(\{0\})=1$ and this implies that $V_{2}=0$ or equivalently that $X$ is a Brownian motion.

Remark. The most important special case of the factorization of Theorem 6 occurs when the underlying random measure has stationary components.

V. Relationships between independence and normality. It is clear from the corollary to Theorem 1 that the independence of $y_{1}, \cdots, y_{n}$ entails restrictions on pairs of random variables while, from the corollary to Theorem 4, normality requires restrictions only on the individual random variables. Consequently it is possible to relate independence to normality only if auxiliary conditions are imposed. The present section is concerned with an investigation of these auxiliary conditions. Our first result represents a natural extension of the earlier work in the case of a finite or denumerable number of independent random variables and is closely related to known results in the integral case $[13$, p. 128].

THEOREM 7. Let $y_{1}, \cdots, y_{n}$ be independent and let at least one of the products $f_{i}(s) f_{k}(s)$, $j \neq k=1, \cdots, n$, be nonvanishing at every point of $R$ with the possible exception of a 
set of $V$-measure zero. Then the underlying random measure is a Brownian motion and the random variables $y_{1}, \cdots, y_{n}$ are normally distributed.

Proof. Denote by $B_{i k}$ the set $\left\{s: f_{i}(s) f_{k}(s) \neq 0\right\}$. Since $y_{j}$ and $y_{k}$ are independent, it follows from Theorem 1 that $V_{2}\left(B_{j k}\right)=0$ for $j \neq k$. Thus $B=\bigcup_{i \neq k} B_{j k}$ is a set of $V_{2}$-measure zero. By hypothesis, the difference $R-B$ is also a set of $V_{2}$-measure zero (since it is a set of $V$-measure zero) so that $V_{2}=0$ which in turn implies that the random measure is a Brownian motion and that the variables $y_{1}, \cdots, y_{n}$ are normally distributed.

As a characterization of the normality of the random variables $y_{1}, \cdots, y_{n}$, Theorem 7 is not completely satisfactory because (i) independence of $y_{1}, \cdots, y_{n}$ is required, a property that is not necessary for their normality, and (ii) normality is achieved as a consequence of the stronger condition that the underlying random measure be a Brownian motion. The next result uses a more suitable independence relationship and does not require that the random measure be a Brownian motion.

THEOREM 8. The random variables $y_{1}, \cdots, y_{n}$ are jointly normal if and only if

$$
f_{i}(s) f_{k}(s)=O\left[V_{2}\right], \quad j \neq k=1, \cdots, n
$$

and for every $j \neq k$ the random variables $\left(\sigma_{i} y_{k}+\sigma_{k} y_{i}\right)$ and $\left(\sigma_{j} y_{k}-\sigma_{k} y_{i}\right)$ are independent where $\sigma_{k}^{2}=E y_{k}^{2}$.

Proof. Since the theorem obviously holds for degenerate random variables, it is no restriction to assume that $y_{1}, \cdots, y_{n}$ are nondegenerate. To establish the necessity observe that if $y_{i}$ and $y_{k}$ are normal, we have, from Theorem $4, f_{j}(s)=f_{k}(s)=O\left[V_{2}\right]$ so that $f_{2}(s) f_{k}(s)=O\left[V_{2}\right]$. Furthermore $\left(\sigma_{j} y_{k}+\sigma_{k} y_{i}\right)$ and $\left(\sigma_{i} y_{k}-\sigma_{k} y_{i}\right)$ are normal, and since they are uncorrelated, they are independent.

Turning to the sufficiency, the independence of $\left(\sigma_{i} y_{k}+\sigma_{k} y_{i}\right)$ and $\left(\sigma_{i} y_{k}-\sigma_{\dot{k}} y_{i}\right)$ implies (from the proof of Theorem 2)

$$
\begin{aligned}
0= & \int_{R} \int_{-\infty}^{\infty}\left[\sigma_{i} f_{k}(s)+\sigma_{k} f_{i}(s)\right]^{2}\left[\sigma_{i} f_{k}(s)-\sigma_{k} f_{i}(s)\right]^{2} \lambda^{2} G_{2}(d s \times d \lambda) \\
= & \sigma_{i}^{4} \int_{R} \int_{-\infty}^{\infty} f_{k}^{4}(s) \lambda^{2} G_{2}(d s \times d \lambda)+\sigma_{k}^{4} \int_{R} \int_{-\infty}^{\infty} f_{i}^{4}(s) \lambda^{2} G_{2}(d s \times d \lambda) \\
& -2 \sigma_{i}^{2} \sigma_{k}^{2} \int_{R} \int_{-\infty}^{\infty} f_{i}^{2}(s) f_{k}^{2}(s) \lambda^{2} G_{2}(d s \times d \lambda) .
\end{aligned}
$$

Because $f_{j}(s) f_{k}(s)=O\left[V_{2}\right]$, the last integral is zero and it follows that $f_{i}(s)=O\left[V_{2}\right]$ and $f_{k}(s)=O\left[V_{2}\right]$ which, by Theorem 4, implies that $y_{i}$ and $y_{k}$ are normally distributed. A similar argument applied to the remaining random variables completes the proof.

The conditions of Theorem 8 can be stated in several equivalent ways. For example, the independence of the random variables $\left(\sigma_{i} y_{k}+\sigma_{k} y_{i}\right)$ and $\left(\sigma_{i} y_{k}-\sigma_{k} y_{i}\right)$ can be expressed in terms of their moments (Theorem 2) or in terms of their conditional moments (Theorem 3). Also from the proof of Theorem 2 it is clear that the requirement $f_{i}(s) f_{k}(s)=$ $O\left[V_{2}\right]$ is equivalent to the condition $E y_{i}^{2} y_{k}^{2}=E y_{i}^{2} \cdot E y_{k}^{2}+2 E^{2} y_{1} y_{k}$.

VI. An application. As an application of the relationship between independence and normality we will consider three different series expansions for the linear processes [14]. With suitable restrictions on the processes, it will be shown that the random coefficients in the expansions are independent only if the processes are normal. Similar 
results have been reported by Pierre [15] who considered a wider class of processes but required stronger assumptions to achieve normality.

Cardinal series expansion. For this example the linear process appears as $y(t)=$ $\int_{-\infty}^{\infty} f(t-s) X(d s)$ where both the integrand and the random measure are real and are defined on $(-\infty, \infty)$. We assume that the measure $V$ is equal to Lebesgue measure on the Borel sets. It follows that the process is wide sense stationary and its correlation function is given by

$$
\begin{aligned}
r(\tau) & =\int_{-\infty}^{\infty} f(\tau+s) f(s) d s \\
& =\frac{1}{2 \pi} \int_{-\infty}^{\infty}|F(\omega)|^{2} \exp [i \omega \tau] d \omega
\end{aligned}
$$

with $F(\omega)$ the Fourier transform of $f(t)$.

If $y(t)$ is bandlimited, that is if its power spectral density vanishes outside the interval $[-\Omega, \Omega]$, we have the well-known expansion $[16, p .11]$

$$
y(t)=\sum_{n=-\infty}^{\infty} y_{n} \frac{\sin \Omega(t-n T)}{\Omega(t-n T)}, \quad \Omega T=\pi
$$

where the series converges in stochastic mean and $y_{n}=y(n T)=\int_{-\infty}^{\infty} f(n T-s) X(d s)$. To avoid trivial cases we will assume that $r(0) \neq 0$.

From (23) it is clear that $f(t)$ is also bandlimited. Suppose that $f(t)$ vanishes on a Borel set of positive (Lebesgue) measure. There is then a nondegenerate interval contained in this set upon which $f(t)$ also vanishes. Consequently $f(t)$ and all its derivatives vanish at some point, which implies that $f(t)$ is identically zero. Since this contradicts the assumption that $r(0) \neq 0$, we conclude that $f(t)$ vanishes at most on a set of measure zero.

Now for any $n \neq m$, it is clear that the product of the integrands $f(n T-s) f(m T-s)$ is also nonvanishing except possibly on a set of measure zero. Thus, if the coefficients $\left\{y_{n}\right\}$ are independent, it follows from Theorem 7 that they are normal and hence $y(t)$ is normal.

Fourier series expansion. In this example we assume that the random measure vanishes outside the interval $[0, T]$ while the integrand $f(t)$ is defined on $(-\infty, \infty)$. The linear process appears as $y(t)=\int_{0}^{T} f(t-s) X(d s)$, and we assume that the measure $V$ is equal to Lebesgue measure on $[0, T]$.

Let $y(t)$ be periodic with period $T$. That is, for every $t$ let $E|y(t)-y(t+T)|^{2}=0$. Because of this periodicity we have

$$
\int_{0}^{T}|f(t-s)-f(t+T-s)|^{2} d s=E|y(t)-y(t+T)|^{2}=0
$$

for every $t$, and it follows that $f(t)$ is almost everywhere equal to a periodic function on $(-\infty, \infty)$. Consequently $y(t)$ is wide sense stationary with correlation function $r(\tau)=\int_{0}^{T} f(\tau+s) f(s) d s$, which is also periodic with period $T$. We further assume that $r(\tau)$ is not a constant function (including the trivial case $r(\tau)=0$ ). For $y(t)$ we then have the Fourier expansion [17, p. 91]

$$
y(t)=y_{0}+2 \sum_{n=1}^{\infty} y_{n}^{(1)} \cos n \omega_{0} t+y_{n}^{(2)} \sin n \omega_{0} t, \quad \omega_{0} T=2 \pi
$$


where convergence is in the stochastic mean with

$$
\begin{aligned}
y_{0} & =\frac{1}{T} \int_{0}^{T} y(t) d t, \\
y_{n}^{(1)} & =\frac{1}{T} \int_{0}^{T} y(t) \cos n \omega_{0} t d t, \quad n=1,2, \cdots, \\
y_{n}^{(2)} & =\frac{1}{T} \int_{0}^{T} y(t) \sin n \omega_{0} t d t, \quad n=1,2, \cdots .
\end{aligned}
$$

Using the periodicity of $f(t)$, we may rewrite the random coefficients

$$
\begin{aligned}
y_{0} & =f_{0} \int_{0}^{T} X(d s), \\
y_{n}^{(1)} & =\int_{0}^{T}\left[f_{n}^{(1)} \cos n \omega_{0} s-f_{n}^{(2)} \sin n \omega_{0} s\right] X(d s), \quad n=1,2, \cdots, \\
y_{n}^{(2)} & =\int_{0}^{T}\left[f_{n}^{(1)} \sin n \omega_{0} s+f_{n}^{(2)} \cos n \omega_{0} s\right] X(d s), \quad n=1,2, \cdots,
\end{aligned}
$$

where $f_{0}, f_{n}^{(1)}$ and $f_{n}^{(2)}$ are the coefficients in the Fourier series representation of $f(t)$. Because $r(\tau)$ is not a constant, at least one of the coefficients $f_{n}^{(1)}$ or $f_{n}^{(2)}$ is nonzero and this, in turn, implies that at least two of the integrands in (26) are nonzero. Clearly these integrands vanish only on a denumerable set and their product also vanishes only on a denumerable set which obviously has measure zero. Thus (Theorem 7 ) if the random coefficients are independent, they must be normal and $y(t)$ is normal.

Karhunen-Loeve expansion. The linear process $y(t)$ is defined as in the first example, and we are concerned with an expansion for $y(t)$ on the interval $[0, T]$. This expansion appears as $[16$, p. 9]

$$
y(t)=\sum_{n=1}^{\infty} y_{n} \varphi_{n}(t), \quad t \in[0, T]
$$

where convergence is again in the stochastic mean and $y_{n}=\int_{0}^{T} y(t) \varphi_{n}(t) d t$. The functions $\varphi_{n}(t)$ are the nonzero solutions of the integral equation $\int_{0}^{T} r\left(t-t^{\prime}\right) \varphi_{n}\left(t^{\prime}\right) d t^{\prime}=\lambda_{n} \varphi_{n}(t)$. It will be assumed that the correlation function $r(\tau)$ is not constant on $[-T, T]$ including the case $r(\tau)=0$.

Rewriting the expression for the random coefficients we have $y_{n}=\int_{-\infty}^{\infty} g_{n}(s) X(d s)$ where $g_{n}(s)=\int_{0}^{T} f(t-s) \varphi_{n}(t) d t$. If $f(t)$ vanishes on a set $B$, then each $g_{n}(s)$ will vanish on the set $N=\{s:[0, T] \subset s+B\}$. There is clearly no contribution to the integral for $y_{n}$ on such a set and it will accordingly be neglected in the following. It follows from Mercer's theorem that $r\left(t-t^{\prime}\right)=\sum_{n=1}^{\infty} \lambda_{n} \varphi_{n}(t) \varphi_{n}\left(t^{\prime}\right)$, convergence being uniform on $[0, T] \times[0, T]$. Now because $r(0) \neq 0$ there is at least one nonzero eigenfunction. Furthermore if there is only one nonzero function, say $\varphi_{n}(t)$, we have $r(0)=\lambda_{n} \varphi_{n}^{2}(t)$ so that $\varphi_{n}(t)$ is constant on $[0, T]$, which implies that $r(\tau)$ is constant on $[-T, T]$ and contradicts the earlier assumption. Consequently there are at least two nonzero functions $\varphi_{n}(t)$ and two nonzero functions $g_{n}(s)$. To proceed further we require knowledge about the zeros of $g_{n}(s)$ and for this it is necessary to impose additional restrictions on the problem.

Assume that the Fourier transform of $f(t)$ is a rational function. Specifically, let $F(\omega)=N(i \omega) / D(i \omega)$ where $N(\cdot)$ and $D(\cdot)$ are polynomials of finite degree, the degree 
of $N(\cdot)$ being strictly less than the degree of $D(\cdot)$. This condition is frequently satisfied in applications, and it is then known that the eigenfunctions satisfy the homogeneous differential equation [17, p. 376]

$$
\lambda_{n} D\left(\frac{d}{d t}\right) D\left(-\frac{d}{d t}\right) \varphi_{n}(t)=N\left(\frac{d}{d t}\right) N\left(-\frac{d}{d t}\right) \varphi_{n}(t), \quad t \in(0, T) .
$$

Similarly it can be shown that $g_{n}(s)$ satisfies the differential equation

$$
D\left(-\frac{d}{d s}\right) g_{n}(s)=N\left(-\frac{d}{d s}\right) \varphi_{n}(s), \quad s \in(0, T) .
$$

Combining these differential equations we obtain

$$
\lambda_{n} D\left(\frac{d}{d s}\right) D^{2}\left(-\frac{d}{d s}\right) g_{n}(s)=N\left(\frac{d}{d s}\right) N\left(-\frac{d}{d s}\right) D\left(-\frac{d}{a s}\right) g_{n}(s), \quad s \in(0, T),
$$

and $g_{n}(s)$ also satisfies a homogeneous differential equation on $(0, T)$. The same procedure shows that $D(-d / d s) g_{n}(s)=0, s \notin[0, T]$, and the general solution for $g_{n}(s)$ is obtained by solving the above equations in their respective regions and properly matching the solutions at the boundaries. Because $F(\omega)$ is a rational function, $f(t)$ has continuous derivatives of all orders except possibly at $t=0$ and the set $N$ is either empty or one of the semi-infinite intervals $(-\infty, 0)$ or $(T, \infty)$. Consequently $g_{n}(s)$ and its derivatives are continuous at every point with the possible exception of $\{0\}$ or $\{T\}$, which are boundary points of $N$.

It is not difficult to show that if $g_{n}(s)$ does not vanish everywhere then, except for the set $N$, it vanishes at most on a set of measure zero (the same argument used for the bandlimited functions of the first example is applicable). Since there are at least two functions $g_{n}(s)$ which do not vanish everywhere, Theorem 7 shows that, if $y_{1}, y_{2}, \cdots$ are independent, they are normal and $y(t)$ is normal.

VII. Acknowledgment. One of the authors wishes to acknowledge the support of the Air Office of Scientific Research under AFOSR Grant 1333-67, of the National Science Foundation under Grant GK-1439, and of the U. S. Army Research Office under Contract DAHC04-69-C-0012.

\section{REFERENCES}

[1] G. Darmois, Analyse generale des liasions stochastiques, Etuder particulière de l'analyse factorielle lineaire, Rev. Inst. Internat. Statistique 21, 2-8 (1953)

[2] V. P. Skitovič, Linear combinations of independent random variables and the normal distribution, Izv. Akad. Nauk SSSR Ser. Mat. 18, 185-200 (1954); English transl., Selected Transl. Math. Stat. and Prob., vol. 2, Amer. Math. Soc., Providence, R. I., 1961, pp. 211-228

[3] C. R. Rao, On some characterizations of the normal law, Sankhyā Ser. A 29, 1-14 (1967)

[4] R. G. Laha, On a characterization of the normal distribution from properties of suitable linear statistice, Ann. Math. Statist. 28, 126-139 (1957)

[5] T. Cacoullos, Characterizations of normality by constant regression of linear slatistics on another linear statistic, Ann Math. Statist. 38, 1894-1898 (1967)

[6] - Some characterizations of normality, Sankhyã Ser. A 29, 399-404 (1967)

[7] R. G. Laha and E. Lukacs, On a linear form whose distribution is identical with that of a monomial, Pacific J. Math 15, 207-214 (1965)

[8] Yu. V. Linnik, Linear forms and the statistical criteria. I, II, Ukrain. Mat. Ž. 5, 207-243, 247-290; (1953); English transl., Selected Transl. Math. Stat. and Prob., vol. 3, Amer. Math. Soc., Providence, R. I., 1963, pp. 1-90 
[9] L. V. Mamal, On the theory of characteristic functions, Vestnik Leningrad. Univ. 15, no. 1, 85-99; (1960), English transl., Selected Transl. Math. Stat. and Prob., vol. 4, Amer. Math. Soc., Providence, R. I., 1963, pp. 153-170

[10] J. Marcinkiewicz, Sur une proprieté de la loi de Gauss, Math. Z. 44, 612-618 (1938)

[11] V. P. Skitovič, On a characterization of Brownian motion, Teor. Verojatnost i Primenen. 1, 361-364 (1956); Theor. Probability Appl. 1, 326-328 (1956)

[12] R. G. Laha and E. Lukacs, On identically disiributed stochastic integrals, Trans. Third Prague Conf. Information Theory, Statist. Decision Functions, Random Processes (Liblice, 1962), Publ. House Czech. Acad. Sci., Prague, 1964, pp. 467-474

[13] E. Lukacs and R. G. Laha, Applications of characteristic functions, Griffin's Statistical Monographs and Courses, no. 14, Hafner, New York, 1964

[14] R. Lugannani and J. B. Thomas, On a class of stochastic processes which are closed under linear transformations, Information and Control 10, 1-21 (1967)

[15] P. A. Pierre, Characteristics of Gaussian random processes by representations in terms of independent random variables, IEEE Trans. Information Theory 15, 648-658 (1969)

[16] A. V. Balakrishnan, Communication theory, McGraw-Hill, New York, 1968

[1i] W. B. Davenport, Jr. and W. L. Root, An introduction to the theory of random signals and noise, McGraw-Hill, New York, 1958 